types jointly influence individuals' STI risk reduction strategies.

Methods We analyzed data from 2011-2017 National Survey of Family Growth for 5,948 and 5,433 unmarried, non-cohabiting sexually active women and men aged 15-44. To describe sexual network position, we created four dichotomous variables that included both past-year number of opposite-sex sex partners (one, multiple) and perceived partner non-monogamy (PPNM, yes/no). Relationship type was defined as a steady or casual sex partner at last sex. Prevalence ratios were used to assess the relationship between network position and two past-year outcomes: condom use at last sex and STI testing. Results were stratified by relationship type.

Results 54\% and 47\% of women and men aged 15-44 had one partner with no PPNM. $6 \%$ and $4 \%$ had one partner with PPNM, 22\% and 29\% had multiple partners with no PPNM and $17 \%$ and $20 \%$ had multiple partners with PPNM. Men with multiple partners and PPNM had the lowest prevalence of condom use of all four groups $(37.7 \%, \mathrm{CI}$ : 33.5, 44.1 compared with 52-56\%). Moreover, women and men in this group with a steady sex partner had higher prevalence of past-year STI testing if they reported PPNM than if they did not (women: $a P R=1.31$; CI: 1.11, 1.55, men: $a P R=1.47 ; C I: 1.19,1.81)$. This same relationship was not seen for women and men whose last sex was with a casual partner.

Conclusion Individuals' STI risk reduction strategies depend on sexual network position and relationship type. Those with PPNM and a steady sex partner may seek STI testing more frequently. Proxy sexual network measures from national surveys may help target STI prevention and testing interventions. Disclosure No significant relationships.

\section{P249 WHAT WORKS IN PARTNER NOTIFICATION FOR SEXUALLY TRANSMITTED INFECTIONS, INCLUDING HIV? SYSTEMATIC REVIEW AND META-ANALYSIS}

${ }^{1}$ Mweete Nglazi, ${ }^{1}$ Taryn Young, ${ }^{2}$ Catherine Mathews, ${ }^{1}$ Moleen Zunza, ${ }^{3}$ Nicola Low*. 'Stellenbosch University, Centre for Evidence-based Health Care, Cape Town, South Africa; ${ }^{2}$ South African Medical Research Council, Health Systems Research Unit, Cape Town, South Africa; ${ }^{3}$ University of Bern, Institute of Social And Preventive Medicine (ISPM), Bern, Switzerland

\subsection{6/sextrans-2019-sti.382}

Background Partner notification (PN) is a core component of the management of sexually transmitted infections (STI). A range of methods can help to find, test and treat sexual partners of an infected person (index patient). The objective of this review was to assess the effects of different PN strategies in people with STI, including HIV infection.

Methods We conducted this review for the Cochrane STI Review Group. We searched for randomised controlled trials (RCTs) in Cochrane, MEDLINE and EMBASE databases, reference lists and trial registries up to 18 October 2018. Two independent reviewers conducted screening, selection and data extraction. Primary biological outcomes were: repeated infection in index cases with curable STI and new infections in partners of people with HIV. We conducted meta-analysis where appropriate.

Results We included 37 trials from 14 countries in Asia, Australia, Europe, South America, sub-Saharan Africa and USA in patients with bacterial STIs or trichomonas $(n=26$ trials), STI syndromes $(n=5)$, HIV $(n=5)$, mixed STI $(n=1)$. The 37 trials assessed 56 comparisons between PN strategies, with the most common being enhanced patient referral $(n=22)$ and expedited partner therapy (EPT, $n=18$ ) for curable STI and contract referral for $\operatorname{HIV}(n=3)$. For index cases with chlamydia, gonorrhoea or trichomonas as a combined outcome, EPT reduced repeat infection compared with simple patient referral $(\mathrm{n}=6,390$, relative risk $0.71,95 \%$ confidence interval, CI 0.59-0.86, I-squared 0\%) but not compared with enhanced patient referral $(n=1,220$, relative risk 0.96 , 95\% CI $0.6-$ 1.53, I-squared 33\%). For HIV, contract referral, versus simple patient referral, increased the number of partners found infected $(n=1,884$, mean difference 0.15 , 95\% CI $0.06-0.24$, I-squared 0\%).

Conclusion EPT is better than simple patient referral, but not better than enhanced patient referral for curable STI. Many PN strategies have limited evidence. Future trials should strengthen evidence of effectiveness by evaluating existing PN strategies using biological outcomes.

Disclosure No significant relationships.

\section{P250 INTERIM ANALYSIS OF INDIVIDUAL RISK FACTORS, SEXUAL NETWORKS, AND STI RISK IN A MILITARY POPULATION}

${ }^{1}$ Nicholas Magno, ${ }^{2}$ Eric Garges*, ${ }^{2}$ Tzucheg Kao, ${ }^{3}$ Realisha Smith, ${ }^{3}$ Tahaniyat Lalani. 'USUHS, Rockville, USA; ${ }^{2}$ Uniformed Services University of the Health Sciences, Department of Preventive Medicine and Biostatistics, Bethesda, USA; ${ }^{3}$ Henry M. Jackson Foundation, Rockville, USA

\subsection{6/sextrans-2019-sti.383}

Background Sexually transmitted infections (STIs) are a consistent medical concern among military service members, who may experience select rates well above their civilian counterparts. Differences in individual risk behaviors as well as network risk factors are different in military populations which may explain the increased rates of select STIs. We performed a pilot study to validate a STI risk assessment survey tool for future use in the military population.

Methods Naval Medical Center Portsmouth was used to pilot a survey for a larger, egocentric social, and sexual network study in military populations. Study participants $(n=50)$ completed an anonymized computer-assisted survey focusing on STI risk factors, including a sexual partner inventory. We evaluated individual demographics, behavioral risk factors, and sexual partnership formation to include mixing patterns and concurrency. Recent history of STI was self-reported in the survey and validated with the participant's medical record. Fischer's exact test was used if applicable. For partners, data was weighted and Wald Chi-square test was used to assess association between variables.

Results Completion of the survey and sexual inventory by respondents was common. The majority were active duty, enlisted, and male. The survey suggested evidence of concurrency and disassortive mixing by age, race, and active duty status. Respondent $(p=0.0089)$ and sexual partner $(p=0.0401)$ alcohol consumption before sex was common and associated with history of STI. Condom use was inconsistent and less frequent with main/steady partners when compared to casual and anonymous partners.

Conclusion The high completion rate demonstrated in this pilot study support that a military population will complete a detailed STI risk index including sexual partner inventory. Interim data analysis suggests that common individual risk 
factors such as network/partnership risk factors can be analyzed using an egocentric survey design. These results will have future implications for targeted prevention programming in the U.S. Military.

Disclosure No significant relationships.

\section{P251 DEVELOPING PARTNER NOTIFICATION OUTCOMES FOR BACTERIAL STI BY SEX-PARTNER TYPE: INTERNATIONAL PERSPECTIVES}

${ }^{1}$ Sonali Wayal, ${ }^{1}$ Tamsin Mckinnon, ${ }^{2}$ Claudia Estcourtt, ${ }^{3}$ Nicola Low, ${ }^{1}$ Catherine Mercer, ${ }^{4}$ Merle Symonds, ${ }^{5}$ Paul Flowers, ${ }^{6}$ Jackie Cassell. ${ }^{1}$ University College London, Institute For Global Health, London, UK; ${ }^{2}$ Glasgow Caledonian University, School of Health and Life Sciences, Glasgow, UK; ${ }^{3}$ University of Bern, Institute of Social and Preventive Medicine (ISPM), Bern, Switzerland; ${ }^{4}$ Barts Health NHS Trust, London, UK; ${ }^{5}$ University of Glasgow, MRC/CSO Social and Public Health Sciences Unit, Glasgow, UK; ${ }^{6}$ Brighton and Sussex Medical School, Primary Care and Public Health, Brighton and Hove, UK

\subsection{6/sextrans-2019-sti.384}

Background Sex-partner type influences sexually transmitted infection (STI) risk. Evaluating partner notification (PN) outcomes by sex-partner type could facilitate effective targeting of resources for PN for STIs. To inform development of PN outcomes for bacterial STIs, we reviewed PN guidelines and randomised control trials (RCTs) for sex-partner type characterisation and its impact on PN outcomes.

Methods We searched online/via experts for PN guidelines worldwide and systematically reviewed RCTs of PN for bacterial STIs in PubMed to December 2018. We extracted data on $\mathrm{PN}$ recommendations and outcomes by sex-partner type.

Results We found PN guidelines from United Kingdom (UK), United States of America (USA), Canada, Australasia, Australia, and New Zealand (NZ). They recommend collecting sex-partner data using terms such as: 'regular'/'main'/'primary'/'casual'/'past'/'anonymous', without providing definitions. Australasian, NZ, Australian, and USA guidelines recommend prioritising $\mathrm{PN}$ based on factors that can enhance STI risk (e.g. having multiple partners), and emphasise PN of 'regular' partners to prevent index case re-infection. Only Australian guidelines outline auditable PN outcomes accounting for sex-partner type: index-reported number of treated 'current regular partners' or 'all past partners (includes current casual partners)'. Ten of 28 RCTs reported study participants' baseline data on sex-partner type (e.g. 'steady'/ 'regular'/'main'/'long-term'/'casual'/'one-time'), without defining them. Three RCTs reported PN outcomes by sex-partner type. Two RCTs reported higher chlamydia/gonorrhoea/trichomonas treatment rates for 'main' than 'casual' partners using expedited-partner-therapy (EPT) vs. patient-referral. Another RCT reported no difference in chlamydia re-infection rates in EPT vs. self-referral among women with a single 'steady' partner than women in overall trial.

Conclusion Current PN guidelines do not define sex-partner type nor address public health benefits of notifying different sex-partners. Sex-partner type definitions should be developed and integrated in clinical practice. RCTs should examine the effect of sex-partner types on PN outcomes. PN guidelines should account for sex-partner type based on evidence from RCTs.

Disclosure No significant relationships.

\section{P252 \\ PREDICTABILITY OF PREVALENCE OF SEXUALLY TRANSMITTED INFECTION ON COMPLEX SEXUAL NETWORK}

${ }^{1}$ Ryosuke Omori ${ }^{\star},{ }^{2}$ Laith Abu-Raddad. ${ }^{1}$ Hokkaido University, Research Center For Zoonosis Control, Sapporo, Japan; ${ }^{2}$ Weill Cornell Medicine-Qatar, Doha, Qatar

\subsection{6/sextrans-2019-sti.385}

Background Estimation of epidemic potential of a sexually transmitted infection (STI) is difficult due to difficulty in measuring and quantifying the sexual network and implications for infection transmission. We demonstrate an approach for predicting the epidemic potential of an STI using data on another STI for men who have sex with men.

Methods An individual-based mathematical model was constructed to describe sex partnering and STI concurrent transmission, namely HIV, herpes simplex virus type 2 (HSV-2), gonorrhea, chlamydia, and syphilis. The model was parameterized with representative biological and behavioral data. 500 heterogeneous sexual networks were simulated, on each of which STI transmission was also simulated. Correlations were assessed on model simulations (STI prevalences). Regressions were conducted to evaluate the predictability of HIV prevalence from each of the other STI prevalences.

Results Across these simulations, Spearman's rank correlation coefficient was 0.46 (95\% CI: $0.37-0.55)$ between HIV and HSV-2, 0.90 (95\% CI: 0.88-0.91) between HIV and gonorrhea, 0.82 (95\% CI: 0.78-0.86) between HIV and chlamydia, 0.82 (95\% CI: 0.78-0.84) between HIV and syphilis, 0.31 (95\% CI: $0.21-0.40$ ) between HSV-2 and gonorrhea, 0.82 (95\% CI: $0.78-0.86$ ) between HSV-2 and chlamydia, 0.15 (95\% CI: $0.05-0.25)$ between HSV-2 and syphilis, $0.70(95 \%$ CI: $0.65-0.75)$ between gonorrhea and chlamydia, $0.93 \quad(95 \%$ CI: 0.92-0.95) between gonorrhea and syphilis, and 0.56 (95\% CI: 0.49-0.61) between chlamydia and syphilis. The adjusted R-squared for predicting HIV prevalence using each individual STI prevalence was 0.40 for HSV-2, 0.77 for gonorrhea, 0.71 for chlamydia, and 0.57 for syphilis. The adjusted R-squared for predicting HIV prevalence in a model that includes all other STI prevalences was 0.92 .

Conclusion STI prevalence is a proxy biomarker of HIV prevalence across heterogeneous sexual networks, explaining a considerable fraction of HIV prevalence variation. However, the strength of the association between each pair of STIs varies across STIs.

Disclosure No significant relationships.

\section{P253 HPV VACCINE KNOWLEDGE AND ACCEPTABILITY AMONG MSM IN LEBANON: A QUALITATIVE STUDY}

${ }^{1}$ Ismael Maatouk*, ${ }^{2}$ Rusi Jaspal. ${ }^{1}$ Clemenceau Medical Center, Dermatology-STIS, Beirut, Lebanon; ${ }^{2}$ De Montfort University, Faculty of Life and Health Sciences, Leicester, UK

\subsection{6/sextrans-2019-sti.386}

Background Some countries specifically recommend HPV vaccination for MSM up to 26 years of age. In Lebanon, free HPV vaccine is not administered. The purpose of this exploratory study was to characterize the knowledge and beliefs of Lebanese MSM regarding HPV vaccination.

Methods Participantsthrough contacts of MSM consulting an STD clinic in Beirut-Lebanon were recruited. In-depth 